\title{
Efeito da desinfecção química nas superfícies de guta-percha e Resilon
}

\author{
Effects of short-term chemical disinfection on gutta-percha and \\ Resilon surfaces
}

\author{
Maíra do Prado* \\ Danielle Ferreira de Assis ${ }^{* *}$ \\ Renata Antoun Simão ${ }^{* * *}$
}

\begin{abstract}
Resumo
Objetivo: Avaliar os efeitos das soluções de hipoclorito de sódio 5,25\% ( $\mathrm{NaOCl}$ ) e clorexidina $2 \%$ (CHX) na topografia e rugosidade de superfícies de guta-percha (GP) e Resilon (RE). Materiais e método: Superfícies planas de guta-percha e Resilon foram utilizadas. Seis grupos foram avaliados: Controle GP (CGP) - guta-percha imersa em água destilada (AD) por 1 minuto; $C H X G P$ (CHXGP) - guta-percha imersa em CHX por 1 minuto e em seguida lavada com $A D ; \mathrm{NaOCl} G P(N G P)$ - guta-percha imersa em $\mathrm{NaOCl}$ por 1 minuto e em seguida lavada com AD; Controle RE (CR) - Resilon imerso em $A D$ por 1 minuto; $C H X R E$ (CHXR) - Resilon imerso em $\mathrm{CHX}$ por 1 minuto e em seguida lavado com $A D ; \mathrm{NaO}-$ $\mathrm{Cl} R E(\mathrm{NR})$ - Resilon imerso em $\mathrm{NaOCl}$ por 1 minuto e em seguida lavado com AD. Microscopia eletrônica de varredura (MEV), Espectroscopia de energia dispersiva (EDS) e Perfilometria foram utilizadas para análise das amostras antes e após os tratamentos. Os valores de rugosidade obtidos por perfilomentria foram avaliados estatisticamente utilizando-se os testes de Kruskal-Wallis e Mann-Whitney $(p<0,05)$. MEV e EDS foram avaliados qualitativamente. Resultados: Os valores inicial e final de rugosidade foram respectivamente: CGP - 0,15 \pm $0,02 / 0,15 \pm 0,01 ; C H X G P-0,16 \pm 0,02 / 0,15 \pm 0,01$; $N G P-0,17 \pm 0,01 / 0,16 \pm 0,02 ; C R-0,16 \pm 0,01 / 0,16$ $\pm 0,01 ; C H X R-0,16 \pm 0,01 / 0,16 \pm 0,01$ e $N R-0,16 \pm$ $0,01 / 0,16 \pm 0,01$. A análise estatística não revelou alterações significativas nos valores de rugosidade após o uso de soluções para descontaminação. Adicionalmente, não foram observadas alterações na topografia e na composição química. Conclusão: A desinfecção química não causou alterações nas superfícies de guta-percha e Resilon.
\end{abstract}

Palavras-chave: Topografia. Irrigantes do canal radicular. Materiais dentários. Endodontia.

\section{Introdução}

O sucesso da terapia endodôntica está associado à prevenção ou à cura das patologias perirradiculares, à ausência de sintomatologia e a sinais clíni$\cos ^{1}$. Nesse sentido, a prevenção da contaminação no sistema de canais radiculares é um importante aspecto da terapia endodôntica ${ }^{2}$.

Durante a obturação, são utilizados cones de guta-percha ou Resilon, como material obturador principal, concomitante ao uso de cimentos endodônticos. Embora tais cones sejam fabricados em condições assépticas, eles podem ser contaminados na armazenagem ou durante seu uso na clínica ${ }^{3,4}$.

Devido às suas características de termoplasticidade, estes materiais não podem ser esterilizados utilizando-se o processo convencional de autoclavagem. Para este fim, soluções com poder antimicrobiano como o hipoclorito de sódio e a clorexidina são utilizadas $^{4,5}$.

O efeito dessas soluções na topografia dos cones vem sendo objeto de estudo há alguns anos. Entretanto, a literatura é ainda bastante controversa nesse aspecto. Estudos utilizando microscopia eletrônica e varredura (MEV) mostram, qualitativamente, não haver modificações topográficas na superfície desses materiais ${ }^{2,4,6,7}$. Ao passo que análises quantitativas, utilizando microscopia de força atômica, relatam modificações nessa topografia ${ }^{8,11}$. 
O perfilômetro é um instrumento utilizado para medir o perfil de diferentes superfícies, com o objetivo de quantificar a rugosidade dessas. Esta técnica tem sido vastamente utilizada em odontologia, principalmente no campo da dentística restauradora, para avaliar a rugosidade de materiais restauradores e da dentina ${ }^{12-14}$. Contudo, não se encontram relatos na literatura utilizando a técnica proposta nesta investigação para análise de materiais obturadores endodônticos.

O objetivo do presente estudo foi avaliar a topografia e a rugosidade de cones de guta-percha e Resilon após desinfecção com soluções de hipoclorito de sódio e clorexidina, por microscopia eletrônica de varredura e perfilometria.

\section{Materiais e método}

Cones medium de guta-percha (Odous De Deus $^{\circledR}$, Belo Horizonte, MG, Brasil) e Resilon (Pentron Clinical Technologies, Wallingford, CT, EUA) foram utilizados na presente pesquisa.

\section{Preparo das amostras}

Os cones foram colocados entre 2 placas de vidro, sobre uma superfície aquecida, a fim de obter-se superfícies planas ( $4 \mathrm{~mm} \times \mathrm{mm}$ ). Um total de 18 superfícies de cada material foram confeccionadas. As amostras foram então lavadas em água destilada e secas com gás de nitrogênio.

Em seguida as amostras foram divididas aleatoriamente nos grupos apresentados na Tabela 1.

Tabela 1 - Grupos experimentais

\begin{tabular}{|c|c|}
\hline Grupos & Materiais/ Tratamentos \\
\hline Controle GP (CGP) $(n=15)^{*}$ & Guta-percha imersa em água destilada por 1 minuto. \\
\hline $\mathrm{CHX}$ GP $(\mathrm{CHXGP})(\mathrm{n}=15)^{*}$ & $\begin{array}{l}\text { Guta-percha imersa em solução de clorexidina } 2 \% \text { por } 1 \text { minuto e em seguida lavada } \\
\text { com } 10 \mathrm{~mL} \text { de água destilada. }\end{array}$ \\
\hline $\mathrm{NaOCl}$ GP (NGP) $(\mathrm{n}=15)^{*}$ & $\begin{array}{l}\text { Guta-percha imersa em solução de } \mathrm{NaOCl} 5,25 \% \text { por } 1 \text { minuto e em seguida lavada } \\
\text { com } 10 \mathrm{~mL} \text { de água destilada. }\end{array}$ \\
\hline Controle RE (CR) $(n=15)^{*}$ & Resilon imerso em água destilada por 1 minuto. \\
\hline $\mathrm{CHX} \operatorname{RE}(\mathrm{CHXR})(\mathrm{n}=15)^{*}$ & $\begin{array}{l}\text { Resilon imerso em solução de clorexidina } 2 \% \text { por } 1 \text { minuto e em seguida lavado com } \\
10 \mathrm{~mL} \text { de água destilada. }\end{array}$ \\
\hline $\mathrm{NaOCI} \operatorname{RE}(\mathrm{NR})(\mathrm{n}=15)^{*}$ & $\begin{array}{l}\text { Resilon imerso em solução de } \mathrm{NaOCl} 5,25 \% \text { por } 1 \text { minuto e em seguida lavado com } 10 \\
\mathrm{~mL} \text { de água destilada. }\end{array}$ \\
\hline
\end{tabular}

número de análises por grupo.

Terminado os tratamentos e lavagem, as superfícies foram secas com gás de nitrogênio e analisadas.

Microscopia eletrônica de varredura foi utilizada para análise qualitativa da topografia, espectroscopia de energia dispersiva (EDS) para análise dos elementos químicos presentes na amostra e perfilometria para análise quantitativa de alterações superficiais na rugosidade, antes e após os diferentes tratamentos.

Para análise por MEV e EDS as amostras foram montadas em stubs, recobertas com ouro e analisadas utilizando-se o microscópio Jeol (JSM 6460 LV, Tokio, Japão). Para cada amostra, um total de cinco fotomicrografias com magnificação de 100x foram realizadas. Em cada fotomicrografia, uma área foi selecionada aleatóriamente e EDS realizado (Figura 1 - área laranja). Foram utilizadas três amostras por grupo, totalizando quinze análises $(n=15)$.
Outras três amostras foram avaliadas quantitativamente, utilizando o perfilômetro Dektak IIA (Bruker, Madison, Winsconsin, EUA). Um total de cinco áreas de $1 \mathrm{~mm}$ foram escaneadas antes e após os diferentes tratamentos. Novamente, um total de quinze áreas foram analisadas por grupo $(n=15)$. Os dados foram avaliados estatisticamente utilizando-se os testes de Kruskal-Wallis e MannWhitney $(p<0,05)$.

\section{Resultados}

Fotomicrografias, representativas de cada grupo experimental, são mostradas na Figura 1. Em todos os casos, guta-percha e Resilon tratados com $\mathrm{NaOCl}$ ou $\mathrm{CHX}$, não se observaram alterações topográficas. 
Controle GP

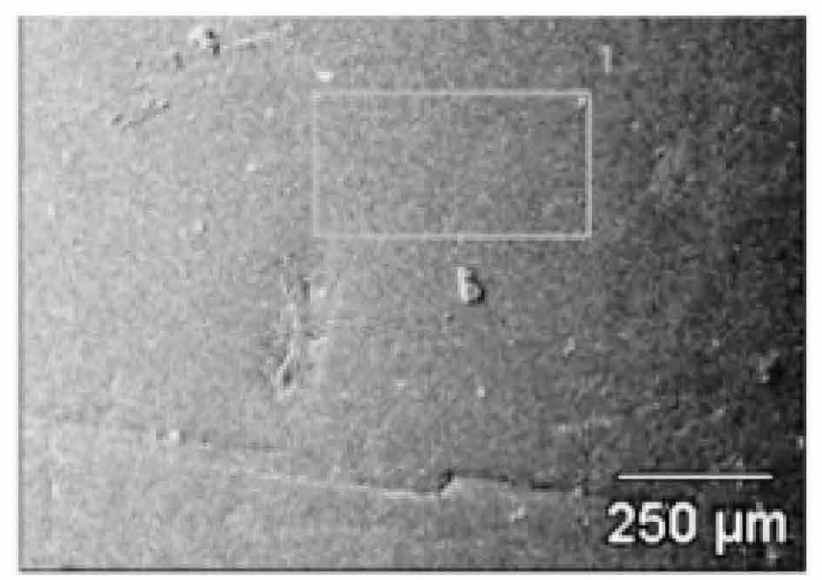

$\mathrm{NaOCl}$ GP

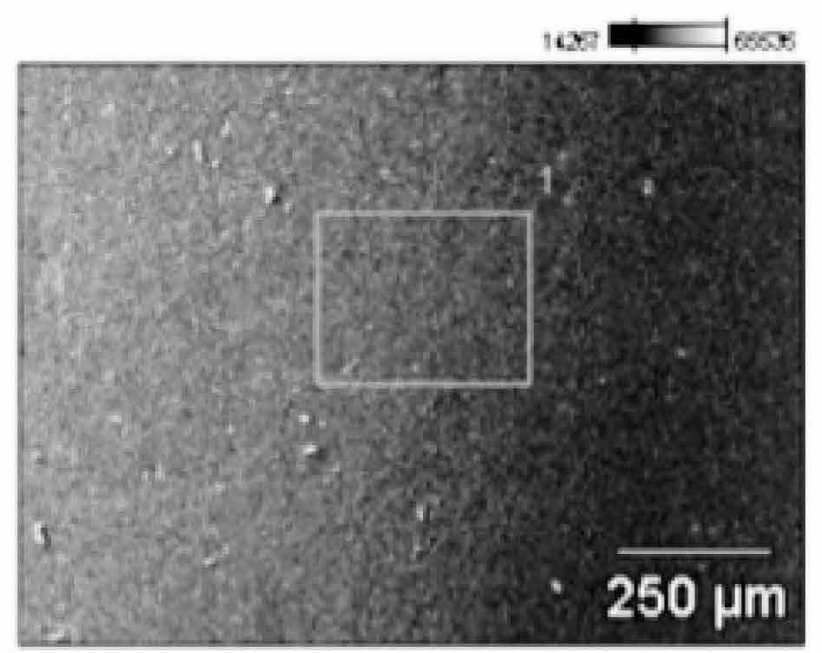

Clorexidina RE

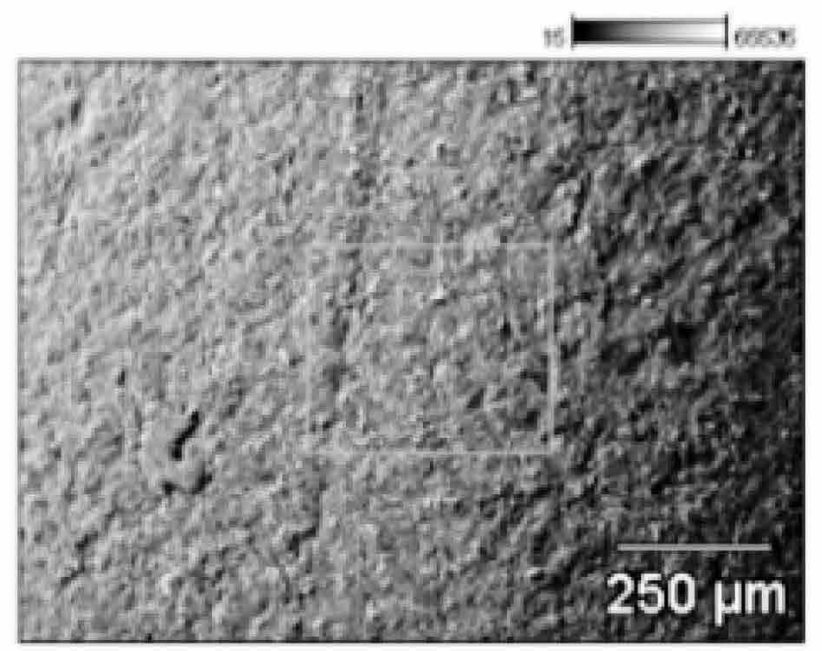

Clorexidina GP

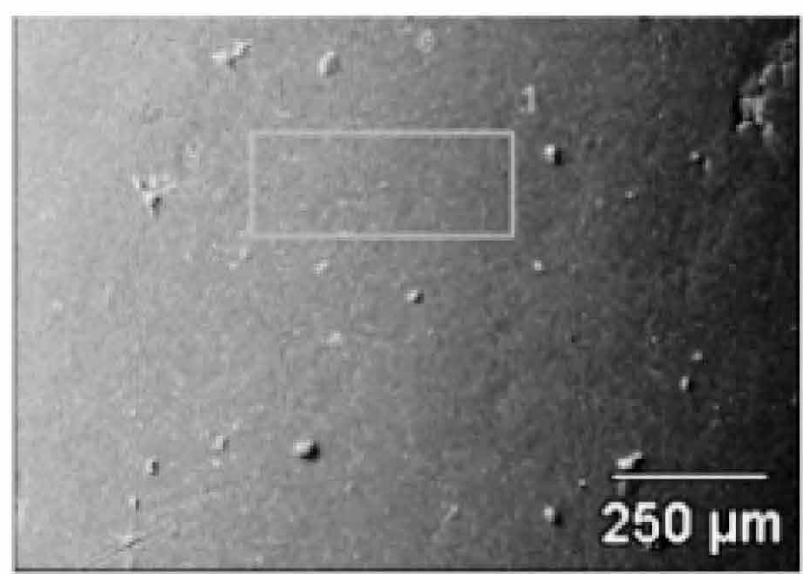

Controle RE

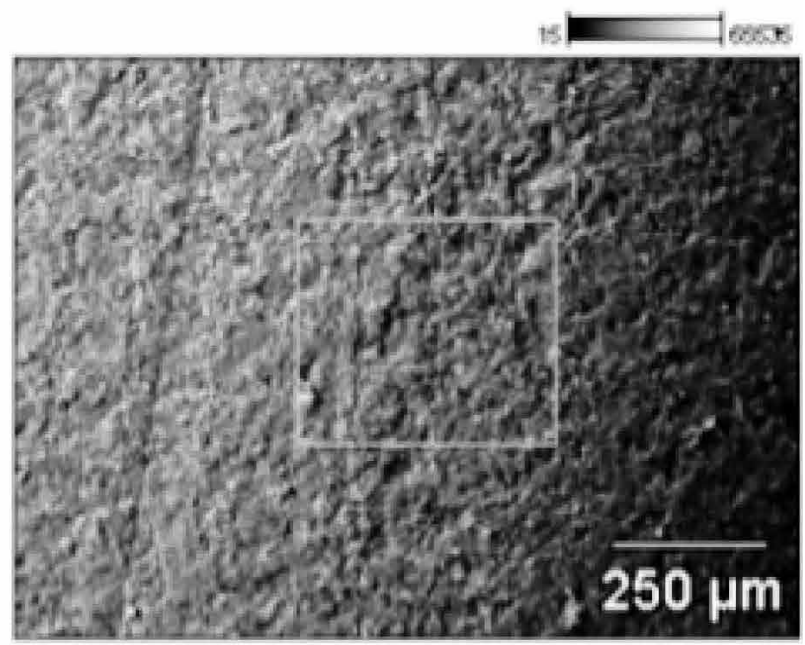

$\mathrm{NaOCl} \mathrm{RE}$

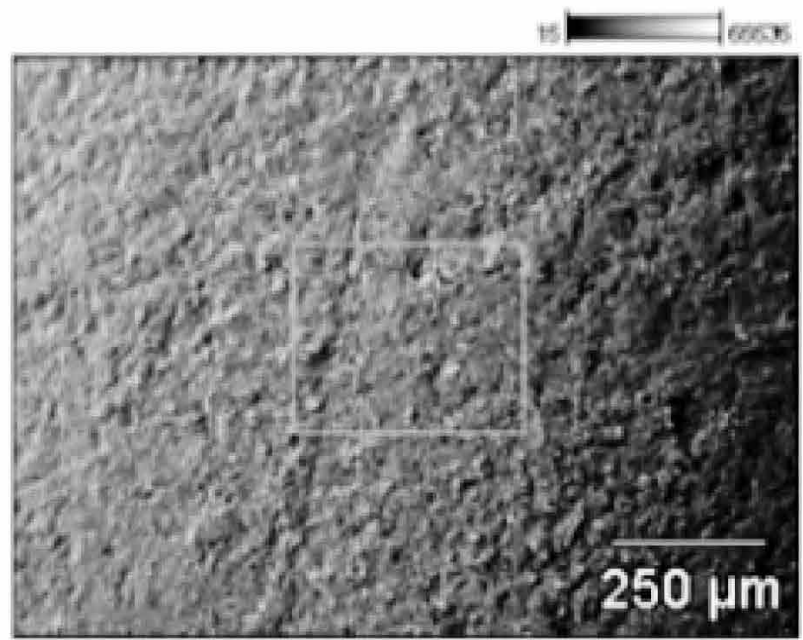

Figura 1 - Fotomicrografia representativa dos diferentes grupos experimentais: controle, clorexidina e NaOCl de guta-percha (GP) e Resilon (RE) 
A Tabela 2 mostra os elementos químicos presentes nas amostras antes e após os diferentes tratamentos. Não se verificou alterações nos componentes químicos nas amostras.

Tabela 2 - Elementos químicos presentes na composição das superfícies de guta-percha e Resilon nos diferentes grupos experimentais

\begin{tabular}{l|c|c|c|c|c|c}
\cline { 2 - 6 } & \multicolumn{3}{c|}{ Gutta-percha } & \multicolumn{3}{c}{ Resilon } \\
\cline { 2 - 7 } & Control & NaOCl & Chlorhexidine & Control & NaOCl & Chlorhexidine \\
\hline $\mathrm{C}$ & $\mathrm{x}$ & $\mathrm{X}$ & $\mathrm{x}$ & $\mathrm{X}$ & $\mathrm{x}$ & $\mathrm{x}$ \\
$\mathrm{O}$ & $\mathrm{x}$ & $\mathrm{X}$ & $\mathrm{x}$ & $\mathrm{X}$ & $\mathrm{x}$ & $\mathrm{x}$ \\
$\mathrm{Zn}$ & $\mathrm{x}$ & $\mathrm{X}$ & $\mathrm{x}$ & & & \\
$\mathrm{Al}$ & $\mathrm{x}$ & $\mathrm{X}$ & $\mathrm{x}$ & & & \\
$\mathrm{Na}$ & & & & $\mathrm{x}$ & $\mathrm{x}$ & $\mathrm{x}$ \\
$\mathrm{Bi}$ & & & & $\mathrm{x}$ & $\mathrm{x}$ & $\mathrm{x}$ \\
$\mathrm{Cl}$ & & & & $\mathrm{x}$ & $\mathrm{x}$ & $\mathrm{x}$ \\
$\mathrm{Ca}$ & & & & $\mathrm{x}$ & $\mathrm{x}$ & $\mathrm{x}$ \\
$\mathrm{Ba}$ & $\mathrm{x}$ & $\mathrm{x}$ & $\mathrm{x}$ & $\mathrm{x}$ & $\mathrm{x}$ & $\mathrm{x}$ \\
$\mathrm{Si}$ & & & & $\mathrm{x}$ & $\mathrm{x}$ & $\mathrm{x}$ \\
\hline
\end{tabular}

A Tabela 3 mostra os valores de rugosidade (RMS) antes e após os diferentes tratamentos. A análise estatística não revelou alterações significativas nos valores de rugosidade após o uso de soluções para descontaminação.

Tabela 3 - Valores de rugosidade RMS (Média \pm Desvio Padrão) dos diferentes grupos experimentais

\begin{tabular}{l|c|c}
\hline \multirow{2}{*}{\multicolumn{1}{c|}{ Grupos }} & \multicolumn{2}{|c}{ Rugosidade $(\mathrm{mm})$} \\
\cline { 2 - 3 } & Inicial & Final \\
\hline Controle GP & $0,15 \pm 0,02$ & $0,15 \pm 0,01$ \\
CHX GP & $0,16 \pm 0,02$ & $0,15 \pm 0,01$ \\
NaOCl GP & $0,17 \pm 0,01$ & $0,16 \pm 0,02$ \\
Controle RE & $0,16 \pm 0,01$ & $0,16 \pm 0,01$ \\
CHX RE & $0,16 \pm 0,01$ & $0,16 \pm 0,01$ \\
NaOCl RE & $0,16 \pm 0,01$ & $0,16 \pm 0,01$ \\
\hline Nota: Sem significância estatistica $(p \geq 0,05)$
\end{tabular}

\section{Discussão}

Embora os cones sejam produzidos sob condições assépticas e as embalagens lacradas possam estar livres de contaminação, esta afirmativa não é valida para todos os casos. Além disso, durante a prática clínica, tanto pelo contato com luvas de procedimentos ou por aerosóis, os cones podem ser contaminados. Por isso, a utilização de soluções descontaminantes previamente à introdução dos mesmos no canal radicular é fundamental ${ }^{2-5,15}$.

$\mathrm{O}$ presente estudo, avaliou qualitativamente, por MEV, o efeito das soluções de $\mathrm{NaOCl} 5,25 \%$ e clorexidina $2 \%$ na topografia dos cones de guta-percha que apresentam uma composição básica de guta-percha (19 a 20\%), óxido de zinco (60 a 75\%), radiopacificadores como o sulfato de bário (1,5 a $17 \%$ ) e outras substâncias como resina, cera e co- rantes (1 a 4\%) ${ }^{16}$ e de Resilon, um polímero sintético (policaprolactano) termoplastificável, que contém vidro bioativo, oxicloridro de bismuto e sulfato de bário (65\% de carga $)^{17}$. Em relação ao tempo de contato com as soluções, no presente estudo avaliou-se a interação durante 1 minuto, por este ser o tempo mínimo necessário para descontaminação de ambos os materiai $\mathrm{s}^{15}$. Cabe ressaltar que a descontaminação está relacionada com o tipo de microrganismos e não com a interação material/solução. Tempos maiores não foram considerados por ser desnecessário em termos de descontaminação. Além disso, o forte poder oxidativo e corrosivo do hipoclorito de sódio poderia causar efeitos na superfície da amostra $^{8,11}$.

Em relação às alterações topográficas na superfície dos materiais devido ao uso das soluções descontaminantes, no presente estudo não foram observadas modificações na topografia. Estes resultados estão de acordo com estudos prévios que avaliaram os efeitos na topografia por $\mathrm{MEV}^{2,4,6}$. Entretanto diferem daqueles que utilizaram como técnica de análise a microscopia de força atômica (AFM) ${ }^{8-11}$. Os resultados divergentes podem estar relacionados as diferenças metodológicas em relação às técnicas de análise. Na microscopia de força atômica a imagem da topografia é gerada a partir da interação entre as forças da sonda (agulha) com a superfície da amostra. Ao passo que na microscopia eletrônica de varredura são emitidos elétrons a partir de um catodo e acelerados através de um anodo. $\mathrm{O}$ feixe de elétrons é focalizado por lentes condensadoras, em um feixe com um ponto focal. Este feixe passa através de bobinas de varredura e placas de deflexão na coluna do microscópio. Quando o feixe primário interage com a amostra, os elétrons perdem energia por dispersão e absorsão em um volume conhecido como volume de interação, o qual se estende de menos de $100 \mathrm{~nm}$ até em torno de $5 \mu \mathrm{m}$ para dentro da superfície da amostra ${ }^{18}$. Comparativamente, devido ao principio de funcionamento das diferentes técnicas pode-se dizer que no MEV uma área mais interna da amostra está sendo avaliada quando comparado ao AFM. O AFM avalia a superfície mais externa da amostra, a última camada. Alterações muito superficiais, que poderiam ser detectadas por AFM, não seriam observadas por MEV por se tratar de uma análise, comparativamente, em maior profundidade. Alterações nanoscópicas vistas no AFM muitas vezes podem não ser detectadas no $\mathrm{MEV}^{19-20}$.

Em relação à análise quantitativa, no presente estudo, alterações na rugosidade superficial, por perfilometria, não foram observadas. Entretanto, prévios estudos utilizando AFM as observaram ${ }^{8-11}$. Novamente, as divergências estão associadas às técnicas de análise. A perfilometria avalia áreas maiores, de $1 \mathrm{~mm}$, ao passo que estudos prévios de AFM avaliaram áreas em torno de $5 \mu \mathrm{m}$. Alterações nanométricas podem não ser percebidas por perfilo- 
metria e serão por AFM pelo fato de ser uma técnica mais sensível para a análise em escala nanométrica.

Em relação a análise química, não se observou alterações na composição química após o uso das soluções desinfetantes. Os componentes encontrados no presente trabalho estão de acordo com estudos prévios que avaliaram a composição química de cones de guta-percha e Resilon ${ }^{2,16,21}$.

Embora modificações topográficas tenham sido previamente relatadas na literatura, no presente estudo utilizando-se perfilometria e MEV, não se observou alterações topográficas, de rugosidade ou química na superfície de guta-percha e Resilon após descontaminação com hipoclorito de sódio 5,25\% e clorexidina $2 \%$. Alterações químicas e topográficas poderiam modificar a interação material obturador/ cimento e desfavorecer o escoamento do cimento no canal e o processo de adesão dos cimentos endodônticos ao material obturador principal (guta-percha ou Resilon).

\section{Conclusão}

A desinfecção química com hipoclorito de sódio e clorexedina não causaram alterações na composição química e na topografia de superfície de guta-percha e Resilon.

\section{Agradecimentos}

Gostaríamos de agradecer pelo suporte técnico à Heleno de Souza da Silva e Jackson Belmiro. À FAPERJ, Capes e CNPq pelo apoio financeiro.

\section{Abstract}

Objective: To assess the effect of 5.25\% sodium hypochlorite $(\mathrm{NaOCl})$ and $2 \%$ chlorhexidine $(\mathrm{CHX})$ solutions on the topography and roughness of gutta-percha and Resilon surfaces. Materials and method: Flat surfaces of gutta-percha and Resilon cones were used. Six groups were assessed: Control GP (CGP) - gutta-percha immersed in distilled water (DW) for 1 minute; $C H X G P$ (CHXGP) - gutta-percha immersed in $C H X$ solution for 1 minute and then washed with DW; $\mathrm{NaOCl} G P(\mathrm{NGP})$ gutta-percha immersed in $\mathrm{NaOCl}$ for 1 minute and then washed with DW; Control RE (CR) - Resilon immersed in DW for 1 minute; CHX RE (CHXR) - Resilon immersed in CHX solution for 1 minute and then washed with $D W$; and $\mathrm{NaOCI} R E(N R)$ - Resilon immersed in $\mathrm{NaOCl}$ for 1 minute and then washed with DW. Scanning Electron Microscopy (SEM), Energy Dispersive Spectroscopy (EDS), and Profilometry were used to analyze the samples before and after treatment. Roughness values obtained through profilometry were statistically analyzed using Kruskal-Wallis e Mann-Whitney tests $(p<0.05)$. SEM and EDS findings were qualitatively assessed. Results: The initial and final roughness values were, respectively: CGP - $0.15 \pm 0.02 / 0.15 \pm 0.01$; CHXGP -
$0.16 \pm 0.02 / 0.15 \pm 0.01 ; N G P-0.17 \pm 0.01 / 0.16 \pm$ $0.02 ; C R-0.16 \pm 0.01 / 0.16 \pm 0.01 ; C H X R-0.16 \pm$ $0.01 / 0.16 \pm 0.01$, and NR $-0.16 \pm 0.01 / 0.16 \pm 0.01$. Statistical analysis revealed no significant changes in roughness values after using decontamination solutions. Additionally, no topographic and chemical changes were observed. Conclusion: Chemical disinfection showed no alteration on gutta-percha and Resilon surfaces.

Keywords: Topography. Root canal irrigants. Dental materials. Endodontics.

\section{Referências}

1. Gutmann JL. Clinical, radiographic, and histologic perspectives on success and failure in endodontics. Dent Clin North Am. 1992; 36(2):379-92.

2. Prado M, Gusman H, Gomes BP, Simão RA. The importance of final rinse after disinfection of gutta-percha and Resilon cones. Oral Surg Oral Med Oral Pathol Oral Radiol Endod. 2011; 111(6):e21-4.

3. da Motta PG, de Figueiredo CBO, Maltos SMM, Nicoli JR, Ribeiro-Sobrinho AP, Maltos KL, et al. Efficacy of chemical sterilization and storage conditions of gutta-percha cones. Int Endod J 2000; 34:435-9.

4. Gomes BPFA, Berber VB, Montagner F, Sena NT, Zaia AA, Ferraz CC, Souza-Filho FJ. Residual effects and surface alterations in disinfected gutta-percha and Resilon cones. $\mathrm{J}$ Endod 2007; 33:948-51.

5. Senia ES, Macarro RV, Mitchell JL, Lewis AG, Thomas L. Rapid sterilization of gutta-percha cones with $5.25 \%$ sodium hypochlorite. J Endod 1975;1:136-40.

6. Short RD, Dorn SO, Kuttler S. The crystallization of sodium hypochlorite on gutta-percha cones after the rapid-sterilization technique: a SEM study. J Endod 2003; 29:670-3.

7. Pang NS, Jung IY, Bae KS, Baek SH, Lee WC, Kum KY. Effects of short-term chemical disinfection of gutta-percha cones: identification of affected microbes and alterations in surface texture and physical properties. J Endod 2007; 33:594-8.

8. Valois CR, Silva LP, Azevedo RB. Effects of $2 \%$ chlorhexidine and $5.25 \%$ sodium hypochlorite on gutta-percha cones studied by atomic force microscopy Int Endod J 2005; 38:425-9.

9. Isci S, Yoldas O, Dumani A. Effects of sodium hypochlorite and chlorhexidine solutions on Resilon (synthetic polymer based root canal filling material) cones: an atomic force microscopy study. J Endod 2006; 32:967-9.

10. Topuz Ö, Sağlam BC, Sen F, Sen S, Gökağaç G, Görgül G. Effects of sodium hypochlorite on gutta-percha and Resilon cones: an atomic force microscopy and scanning electron microscopy study. Oral Surg Oral Med Oral Pathol Oral Radiol Endod. 2011; 112(4):e21-6.

11. Prado M, Gusman H, Gomes BP, Simão RA. Effect of disinfectant solutions on gutta-percha and resilon cones. Microsc Res Tech. 2012; 75(6):791-5.

12. Roberts MJ, Söderholm KJ. Comparison of three techniques for measuring wear of dental restorations. Acta Odontol Scand. 1989; 47(6):367-74.

13. Ozdemir-Ozenen D, Sungurtekin E, Issever H, Sandalli N. Surface roughness of fluoride-releasing restorative materials after topical fluoride application. Eur J Paediatr Dent. 2013; 14(1):68-72. 
14. Cavalli V, Arrais CA, Giannini M, Ambrosano GM. Highconcentrated carbamide peroxide bleaching agents effects on enamel surface. J Oral Rehabil. 2004; 31(2):155-9.

15. Gomes BPFA, Vianna ME, Matsumoto CU, Rossi-Vde P, Zai AA, Ferraz CC, et al. Disinfection of gutta-percha cones with chlorhexidine and sodium hypochlorite. Oral Surg Oral Med Oral Pathol Oral Radiol Endod 2005; 100:512-7.

16. Gurgel-Filho ED1, Andrade Feitosa JP, Teixeira FB, Monteiro de Paula RC, Araújo Silva JB, Souza-Filho FJ. Chemical and X-ray analyses of five brands of dental gutta-percha cone. Int Endod J. 2003; 36(4):302-7.

17. Pawinska M, Kierklo A, Marczuk-Kolada G. New technology in endodontics--the RESILON-Epiphany system for obturation of root canals. Adv Med Science 2006; 51:154-7.

18. Kestenbach HJ, Nocite NCPS, Loos J, Petermann J. Resolução lamelar num novo microscópio eletrônico de varredura. Polímeros 1997; 7(1):58-66.

19. Poletti G, Orsini F, Lenardi C, Barborini E. A comparative study between AFM and SEM imaging on human scalp hair. J Microsc. 2003; 211(3):249-55.

20. Prado M, Gusman H, Gomes BP, Simão RA. A Comparative Study of the Smear Layer Removal Using Scanning Electronic Microscopy and Atomic Force Microscopy. JAMR 2012; 7:21-5.

21. Elzubair A, Elias CN, Suarez JC, Lopes HP, Vieira MV. The physical characterization of a thermoplastic polymer for endodontic obturation. J Dent. 2006; 34(10):784-9.

Endereço para correspondência:

Maíra do Prado

Cid. Universitária-Centro de Tecnologia - Bloco F, sala F-211 - Ilha do Fundão

Caixa Postal 68505

21941-972 - Rio de Janeiro / RJ

Fones: (21) 2562-8526, (21) 2562-8527

E-mail: maira@metalmat.ufrj.br

Recebido: 06/11/2013. Aceito: 23/03/2014. 